01

\title{
Коэффициенты самоуширения и сдвига линий в полосе первого обертона молекулы $\mathrm{HBr}$
}

\author{
(C) P.E. Асффин ${ }^{1}$, А. Доманская ${ }^{2}$, К. Мауль ${ }^{3}$ \\ ${ }^{1}$ Санкт-Петербургский государственный университет, \\ 199034 Санкт-Петербург, Россия \\ ${ }^{2}$ Institute of Physical Chemistry, University of Göttingen, \\ 37077 Göttingen, Germany \\ ${ }^{3}$ Institut für Physikalische und Theoretische Chemie, Technische Universität \\ 38106 Braunschweig, Germany \\ e-mail: r.asfin@spbu.ru
}

Поступила в редакцию 04.06.2021 г.

В окончательной редакции 06.07.2021 г.

Принята к публикации 11.07.2021 г.

\begin{abstract}
Зарегистрированы спектры $\mathrm{HBr}$ с естественным содержанием изотопологов в области первого обертона при комнатной температуре при различных субатмосферных давлениях. Найдены коэффициенты самоуширения и сдвига линий двух изотопологов $\mathrm{HBr}$. И коэффициенты сдвига, и коэффициенты уширения совпадают для разных изотопологов в пределах погрешности. Коэффициенты уширения находятся в хорошем согласии с измеренными ранее, но имеют меньшую погрешность, порядка $1 \%$. Коэффициенты сдвига публикуются впервые. Полученные интенсивности линий обоих изотопологов находятся в удовлетворительном согласии с опубликованными ранее значениями.
\end{abstract}

Ключевые слова: самосдвиг, самоуширение, обертон, галоидоводороды, двухатомные молекулы.

DOI: $10.21883 /$ OS.2021.12.51731.2383-21

\section{Введение}

Бромоводород $(\mathrm{HBr})$ относится к хорошо изученной группе молекул, галоидоводородов (HHal), хотя именно эта молекула исследовалась меньше всего. В ближайшем будущем это может стать недостатком, поскольку спрос на бромоводород из-за его использования в электронике (травление, в батарейках и пр.), согласно исследованию рынка, будет стремительно расти [1,2]. Методы оптического детектирования и мониторинга, например, прямое поглощение лазерного излучения (DLAS), часто требуют знания электрооптических параметров, необходимых для спектрального моделирования. Такие параметры для чистого газа являются предметом обсуждения настоящей работы. Первое измерение коэффициентов самоуширения и интенсивностей в фундаментальной полосе, выполненное в работе [3], было основано на методе кривой роста, применённым к изотопно-неразрешенному спектру. Целью работы [4], в которой впервые были разрешены линии различных изотопологов, было измерение интенсивностей в обертоне, но также была дана оценка коэффициентов уширения. В работе [5] также выполнено измерение этих коэффициентов в $R$-ветви фундаментальной полосы с более высоким разрешением и продемонстрировано, что упомянутые величины для изотопологов $\mathrm{H}^{79} \mathrm{Br}$ и $\mathrm{H}^{81} \mathrm{Br}$ имеют, как и ожидалось, практически одинаковые значения. Дальнейшие исследования первого обертона включают в себя работу [6] с измерениями самоуширения линий $R_{2}(7)$ и $P_{2}(2)^{1}$ в спектре $\mathrm{H}^{79} \mathrm{Br}$ при помощи диодной лазерной спектроскопии с использованием перестраиваемых диодных лазеров (TDLAS), а также работу [7] с набором коэффициентов самоуширения, полученных в качестве побочного продукта при изучении спектральных параметров $\mathrm{HBr}$, возмущенного благородными газами.

B базе данных HITRAN [8,9] коэффициенты самоуширения для всех колебательных полос были взяты из экспериментальных данных [5] для фундаментальной полосы без учета их зависимости от колебательного возбуждения, какой бы слабой она не была. При этом коэффициенты $P$-ветви получены зеркальным отражением значений в $R$-ветви. Интенсивности линий рассчитаны с помощью функции дипольного момента, определенной по имеющимся экспериментальным данным [10]. Помимо упоминавшихся выше работ, интенсивности линий в первом обертоне были измерены Bernage и Niay [11], использовавших метод эквивалентных ширин для разных длин поглощения путей. Интенсивности линий в первом обертоне были измерены, в частности, с целью определения параметров функции дипольного момента [10] в работе [12]. Насколько нам известно, измерения сдвигов линий давлением чистого газа ранее не производилось.

\footnotetext{
${ }^{1}$ Мы используем следующее обозначение для спектральных линий: буквы $R, P$ означают принадлежность к $R$ - или $P$-ветви, целочисленный нижний индекс означает колебательное квантовое число конечного состояния, целое число в скобках - вращательное квантовое число начального состояния.
} 
В настоящей работе мы приводим коэффициенты самоуширения и самосдвига, а также интенсивности линий в первом обертоне чистого газа $\mathrm{HBr}$ при комнатной температуре и субатмосферных давлениях.

\section{Эксперимент и анализ}

\section{Измерения}

Спектры были зарегистрированы на спектрометре Bruker IFS-120 HR Технического университета Брауншвейга, оснащенного светоделителем $\mathrm{CaF}_{2} / \mathrm{Si}$, охлаждаемым жидким азотом приемником LN InSb и вольфрамовой лампой в качестве источника излучения. В данной модели спектрометра запись интерферограммы и фурье-преобразование производились на одной из плат самого спектрометра с довольно жестким ограничением на размер массива. Для достижения высокого разрешения был использован полосной оптический фильтр с окном прозрачности в области $5100-4700 \mathrm{~cm}^{-1}$. Дополнительным плюсом стало то, что автоматически улучшилось отношение сигнал/шум. Газ $\mathrm{HBr}$ (Linde Gas AG, чистота 4.5) напускался в стеклянную кювету длиной $25 \mathrm{~cm} \mathrm{c}$ окнами из $\mathrm{CaF}_{2}$. Окна крепились к фланцам кюветы при помощи эпоксидной смолы Torr Seal ${ }^{\circledR}$. Давление образцов измерялось емкостным манометром MKS Baratron (заявленная погрешность 0.1\% от показываемого значения), работающего в диапазоне 0.1-1000 mbar. Bсе спектры были зарегистрированы при температуре $T=24 \pm 1^{\circ} \mathrm{C}(297 \mathrm{~K})$.

Для каждого давления регистрировались несколько спектров, каждый из которых, в свою очередь, был получен фурье-преобразованием 50 усредненных интерферограмм. Такая процедура помогает оценить стабильность образца. Если спектры оставались неизменными в течение эксперимента, то они усреднялись перед дальнейшей обработкой. При регистрации спектров использовалась прямоугольная функция аподизации. Основные экспериментальные параметры приведены в табл. 1.

\section{Обработка данных}

Спектральные параметры были получены линейной подгонкой. Особенности процедуры подгонки описаны в $[13,14]$. Поскольку газообразный $\mathrm{HBr}$ в природе существует в двух изотопных модификациях, $\mathrm{H}^{79} \mathrm{Br}$ и $\mathrm{H}^{81} \mathrm{Br}(50.7 \%$ и $49.3 \%$ соответственно), то колебательновращательный спектр состоит из дублетов для каждого номера линий $m^{2}$ (рис. 1). Обе компоненты дублета были описаны контуром Фойгта в интервале 60 полных ширин, центрированным посередине между компонентами. Обработка осуществлялась в программе Origin 9.0 с использованием встроенных средств для нелинейной подгонки кривых, дополненных скриптом на языке LabTalk

\footnotetext{
${ }^{2} m=-J$ для $P$-ветви, $m=J+1$ для $R$-ветви, где $J-$ вращательное квантовое число начального состояния.
}

Таблица 1. Экспериментальные параметры: давление образца (непосредственное показание манометра, см. текст), номинальное спектральное разрешение, число сканирований и размер апертуры спектрометра Bruker 120 HR (диаметр)

\begin{tabular}{c|c|c|c|c}
\hline $\mathrm{N}$ & $\begin{array}{c}\text { Давление, } \\
\mathrm{mbar}\end{array}$ & $\begin{array}{c}\text { Разрешение, } \\
\mathrm{cm}^{-1}\end{array}$ & Число сканов & $\begin{array}{c}\text { Апертура, } \\
\mathrm{mm}\end{array}$ \\
\hline 1 & 42.5 & 0.0035 & 500 & 0.8 \\
2 & 103.4 & 0.003 & 250 & 0.8 \\
3 & 222.2 & 0.003 & 350 & 0.8 \\
4 & 290 & 0.003 & 500 & 0.8 \\
5 & 405 & 0.003 & 450 & 0.8 \\
6 & 616 & 0.005 & 500 & 1.0 \\
7 & 706 & 0.005 & 500 & 1.0 \\
8 & 870 & 0.005 & 500 & 1.0
\end{tabular}

для автоматического выбора спектральных интервалов, инициализации параметров и сбора результатов. Во время обработки мы не обнаружили никаких следов влияния аппаратной функции на форму линий.

Доплеровская полуширина контура Фойгта не варьировалась и была приравнена теоретическому значению:

$$
\Delta v_{D}=\frac{v_{0}}{c} \sqrt{\frac{2 \ln 2 k_{B} T}{M_{u} M}},
$$

где $v_{0}$ - волновое число центра линии в $\mathrm{cm}^{-1}, T-$ температура газа в кельвинах (297 К в нашем случае), $M$ - масса молекулы в атомных единицах массы ати $\left(M=80\right.$ для $\mathrm{H}^{79} \mathrm{Br}$ и $M=82$ для $\left.\mathrm{H}^{81} \mathrm{Br}\right), c$ - скорость света, $k_{B}$ - постоянная Больцмана, $M_{u}$ - атомная единица массы. Типичное значение $\Delta v_{D}$ для $\mathrm{HBr}$ в области первого обертона составляет $0.0035 \mathrm{~cm}^{-1}$.

Зависимости площадей, ширин и центров линий от давления для каждого $m$ были аппроксимированы прямыми линиями с помощью Origin. Наклон в линейной регрессии полуширин и волновых чисел центров линий дают соответственно коэффициенты уширения и сдвига. Анализ площадей линий позволяет проверить, есть ли систематическая ошибка в показаниях манометра. Очевидно, что интенсивность всех линий должна быть нулевой при нулевом давлении. Однако точка пересечения в линейной регрессии площадей линий от давления в данной работе была несколько меньше 0 . Коррекция показаний манометра на 2.1 mbar привела к исчезновению ненулевых пересечений с точностью до погрешности манометра. Такая поправка не влияет на наклон регрессий.

На рис. 1 показаны спектры $\mathrm{HBr}$ в области линий $P_{2}(10)$ и $R_{2}(2)$. С увеличением давления интенсивность линий растет, и они уширяются. Видно, что при давлениях выше определенного значения оптическая плотность максимумов линий не меняется: рост интенсивности компенсируется уширением линий. В далеких крыльях полосы (линии с большими $|m|$ ) хорошо выражен сдвиг максимумов полос к низким волновым числам. 

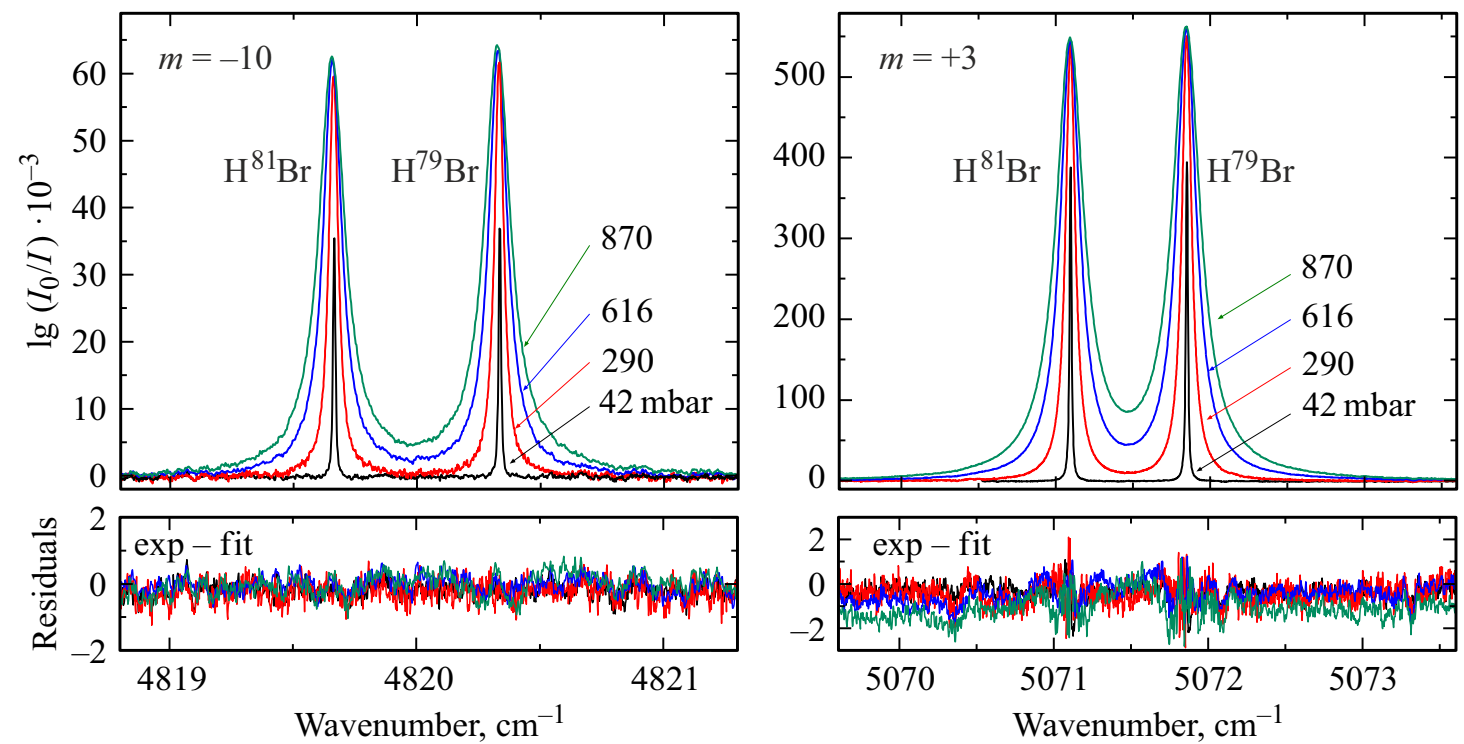

Рис. 1. Эволюция формы линий $P_{2}(10)$ и $R_{2}(2)$ с изменением давления. На нижних панелях показаны разности экспериментальных и полученных подгонкой (фойгтовских) спектров.

В нижней части рис. 1 показаны разности между зарегистрированным и подогнанным спектрами. Отличие между спектрами для линий с большими $J$ незаметно на фоне шума. Однако для линий, близких к началу полосы, вблизи центров линий видны систематические отклонения, вызванные, возможно, сверхтонкой структурой. Похожие отклонения от симметричного контура наблюдались для НІ (рис. 3 в [15]). Константы ядерного квадрупольного взаимодействия для $\mathrm{HBr}$ более чем в 3 раза меньше, чем константы для НІ [15], что приводит к более компактной структуре расщепления с порядком величины $10^{-3} \mathrm{~cm}^{-1}$ [6], тогда как сверхтонкое расщепление в НІ имеет порядок $10^{-2} \mathrm{~cm}^{-1}$. Также возможно, что данные отклонения вызваны отличием профиля экспериментальных линий от контура Фойгта за счет различных малых эффектов, влияющих на профили линий. В любом случае максимальная величина отклонений не превышает $0.5 \%$ от максимума линии.

\section{Результаты и обсуждение}

\section{Уширение}

Зависимости лоренцевых ширин (HWHM) от давления были аппроксимированы прямыми линиями. Коэффициенты самоуширения - являются коэффициентами наклона в соответствующих линейных регрессиях. Экспериментальные данные позволили провести подгонку с высокой точностью (коэффициент детерминации $R^{2}$ для большинства линий превышал 0.99975), за исключением линий с $m>+9$ из-за высокого уровня шума в этой области. Несколько примеров таких аппроксимаций показаны на рис. 2.

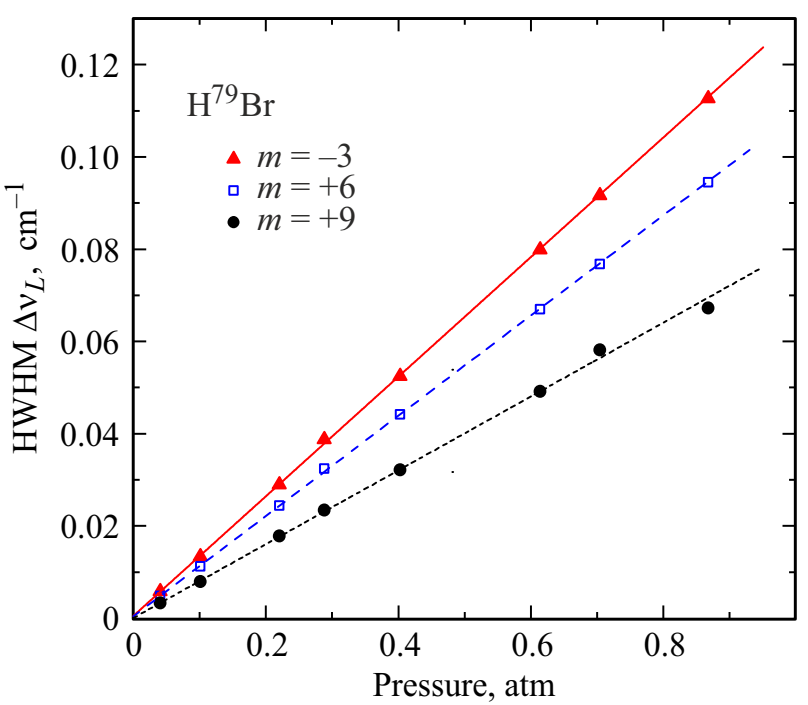

Рис. 2. Несколько примеров зависимости лоренцевой полуширины линий $P_{2}(3), R_{2}(5)$ и $R_{2}(8)$ (номера линий $m=-3,+6$ и +9$)$ от давления, полученных в данной работе.

Грубая оценка влияния масс на коэффициенты уширения $\gamma$, основанная на кинетической теории газов для случая твердых сфер, приводит к следующей зависимости: $\gamma \sim \frac{\sqrt{M_{U}}}{\mu}$, где $M_{U}-$ масса поглощающей молекулы, а $\mu$ - приведенная масса поглощающей и возмущающей молекул [16]. Для систем $\mathrm{H}^{79} \mathrm{Br}$ и $\mathrm{H}^{81} \mathrm{Br}$ она предсказывает различие примерно в $1 \%$, при этом коэффициенты уширения $\mathrm{H}^{79} \mathrm{Br}$ должны быть больше. Это различие близко к погрешности полученных коэффициентов уширения, хотя, возможно, что коэффициенты уширения 
Таблица 2. Коэффициенты самоуширения $\gamma$ $\left(10^{-3} \mathrm{~cm}^{-1} \mathrm{~atm}^{-1}\right)$ и сдвига $\delta\left(10^{-3} \mathrm{~cm}^{-1} \mathrm{~atm}^{-1}\right)$ для первого обертона $\mathrm{HBr}$ при $297 \mathrm{~K}$; среднее значение это средневзвешенное от параметров линий изотопологов; в скобках приведены погрешности, соответствующие доверительной вероятности $95 \%{ }^{\text {a }}$

\begin{tabular}{c|c|c|c|c|c|c}
\hline$m$ & \multicolumn{2}{|c|}{$\begin{array}{r}\text { Коэффициенты уширения, } \\
\gamma\left(10^{-3} \mathrm{~cm}^{-1} \mathrm{~atm}^{-1}\right)\end{array}$} & \multicolumn{2}{|c}{\begin{tabular}{r} 
Коэффициенты сдвига, \\
\multicolumn{2}{|c|}{$\left.\mathrm{cm}^{-3} \mathrm{~atm}^{-1}\right)$}
\end{tabular}} \\
\hline & $\mathrm{H}^{79} \mathrm{Br}$ & $\mathrm{H}^{81} \mathrm{Br}$ & Среднее & $\mathrm{H}^{79} \mathrm{Br}$ & $\mathrm{H}^{81} \mathrm{Br}$ & Среднее \\
\hline-11 & $74(2)$ & $73(2)$ & $73(2)$ & $-5(4)$ & $-6(2)$ & $-5(2)$ \\
-10 & $75(1)$ & $75(1)$ & $74.7(8)$ & $-11(1)$ & $-11.5(8)$ & $-11.4(7)$ \\
-9 & $82(1)$ & $81.6(10)$ & $81.7(7)$ & $-4.2(4)$ & $-4.3(3)$ & $-4.3(2)$ \\
-8 & $89(2)$ & $89(2)$ & $88.8(10)$ & $-10.7(5)$ & $-10.8(7)$ & $-10.7(4)$ \\
-7 & $98(2)$ & $97(2)$ & $98(1)$ & $-5.3(4)$ & $-4.8(3)$ & $-5.0(4)$ \\
-6 & $110(2)$ & $110(2)$ & $110(1)$ & $-9.7(6)$ & $-9.4(4)$ & $-9.5(4)$ \\
-5 & $122(2)$ & $122(2)$ & $122(1)$ & $-7.8(3)$ & $-7.6(6)$ & $-7.7(3)$ \\
-4 & $129(2)$ & $128(2)$ & $129(1)$ & $-8.3(5)$ & $-8.3(9)$ & $-8.3(5)$ \\
-3 & $131(2)$ & $131(2)$ & $131(2)$ & $-2.0(5)$ & $-1.8(7)$ & $-1.9(4)$ \\
-2 & $131(2)$ & $131(2)$ & $131(2)$ & $+2.4(5)$ & $+2.3(6)$ & $+2.3(4)$ \\
-1 & $129(2)$ & $130(2)$ & $129(2)$ & $-6.8(4)$ & $-7.1(7)$ & $-6.8(4)$ \\
1 & $127(2)$ & $127(2)$ & $127(2)$ & $+4.4(7)$ & $+4.7(7)$ & $+4.5(5)$ \\
2 & $126(2)$ & $126(2)$ & $126(1)$ & $-4.7(9)$ & $-4.9(7)$ & $-4.8(6)$ \\
3 & $127(2)$ & $126(2)$ & $127(1)$ & $-4.5(2)$ & $-5.1(6)$ & $-4.6(3)$ \\
4 & $125(2)$ & $124(2)$ & $125(1)$ & $-6.2(4)$ & $-6.6(7)$ & $-6.3(4)$ \\
5 & $118(2)$ & $119(2)$ & $119(1)$ & $-9.1(6)$ & $-8.4(8)$ & $-8.8(6)$ \\
6 & $110(1)$ & $111(2)$ & $110(1)$ & $-8(1)$ & $-9.2(2)$ & $-9.2(3)$ \\
7 & $100(2)$ & $99(2)$ & $99.7(10)$ & $-13(2)$ & $-13.5(6)$ & $-13.4(5)$ \\
8 & $90.2(8)$ & $90(1)$ & $90.3(7)$ & $-11(2)$ & $-12(2)$ & $-11(2)$ \\
9 & $81(3)$ & $82(3)$ & $82(2)$ & $-15(3)$ & $-17(3)$ & $-15(2)$ \\
10 & $78(5)$ & $74(5)$ & $76(4)$ & $-15(3)$ & $-17(3)$ & $-16(3)$ \\
11 & $82(9)$ & $79(8)$ & $81(6)$ & $-23(9)$ & $-18(5)$ & $-19(5)$
\end{tabular}

Примечание. ${ }^{\text {а } О г р а н и ч е н н ы и ̆ ~ н а б о р ~ д а н н ы х ~ д л я ~ к а ж д о г о ~ п а р а м е т р а ~}$ был учтен с использованием значений критерия Стьюдента ( $t$-критерия), см. текст.

для более легкого изотополога действительно несколько больше (табл. 2, вторая и третья колонки).

Чтобы проверить, реальна ли разница между различными изотопологами в наших измерениях, мы рассчитали метрологическую совместимость $f[14,17]$ :

$$
f=\frac{1}{\sqrt{2}} \frac{X_{1}-X_{2}}{\sqrt{\Delta X_{1}^{2}+\Delta X_{2}^{2}}},
$$

где $X_{1}$ и $X_{2}$ - это значения, которые необходимо сравнить, а их погрешности - это $\Delta X_{1}$ и $\Delta X_{2}$. Если $|f|<1$, то величины $X_{1}$ и $X_{2}$ совместимы.

Результаты показаны в нижней части рис. 3. Видно, что коэффициенты уширения для $\mathrm{H}^{79} \mathrm{Br}$ и $\mathrm{H}^{81} \mathrm{Br}$ совпадают с доверительной вероятностью 95\%. Сходные выводы были сделаны и в предыдущих исследованиях [5,7]. Поэтому мы усреднили коэффициенты самоуширения изотопологов, принимая во внимание дисперсии их значений. Полученные результаты также приведены в табл. 2.

На рис. 3 представлены наши результаты наряду с литературными значениями для полосы обертона $[6,8,9]$ и фундаментальной полосы [3,5]. Видно, что коэффициенты уширения сильно зависят от вращательного состояния, однако имеют исчезающе малую зависимость от колебательного возбуждения. Напомним, что коэффициенты уширения из HITRAN для первого обертона скопированы из фундаментальной полосы еще в 1992 г. [18] без учета их колебательной зависимости, и с тех пор остаются неизменными.

Наши результаты для обертона несколько меньше, чем для фундаментальной полосы в центре полосы, но имеют тенденцию к стабилизации на несколько бо́льших значениях в крыльях. Коэффициент уширения для $m=11$, очевидно, является выбросом из-за низкого отношения сигнал/шум в этой области.

Значение для $P_{2}(2)$, полученное в [6], примерно на 9\% меньше, чем наш результат. Упоминание о значительном отличии этого коэффициента уширения было сделано самими авторами, сообщившими о $14 \%$-ном отличии от значения из HITRAN в фундаментальной полосе [6], которое они отнесли, хотя и не окончательно, к искажениям формы линии из-за сверхтонкой структуры. Мы не согласны с этой интерпретацией, поскольку некоторые из наших спектров зарегистрированы в сходных услови-
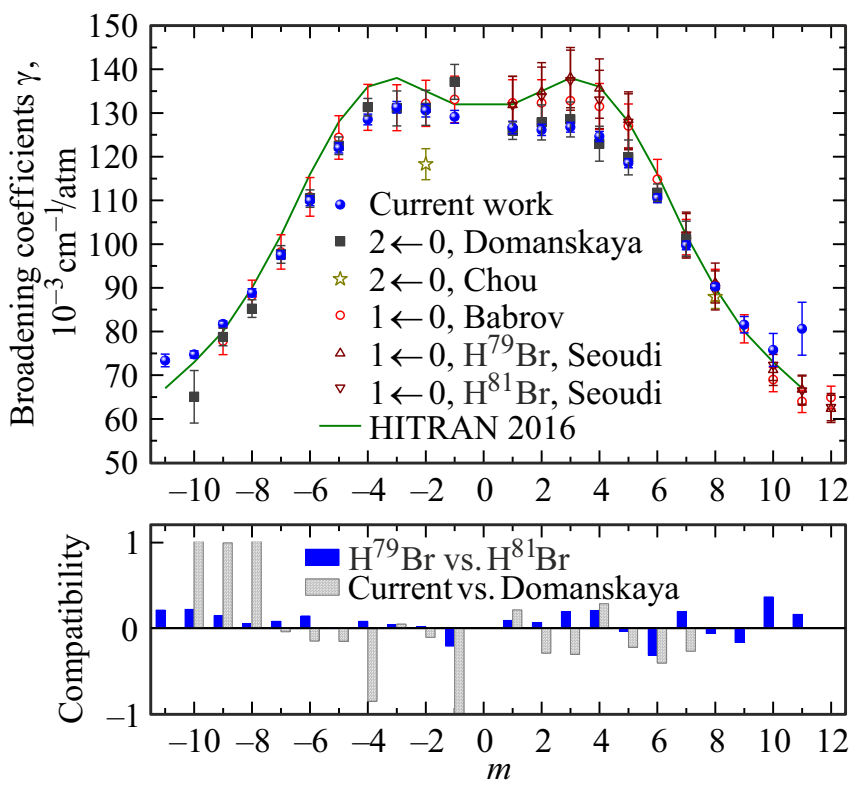

Рис. 3. Зависимость коэффициентов самоуширения $\gamma \mathrm{HBr}$ от номера линии $m$. Результаты данной работы для первого обертона показаны синими кружками. Также представлены более старые данные: Domanskaya et al. [7] (черные квадраты) и Chou et al. [6] (звездочки). Коэффициенты уширения для фундаментального перехода взяты из работ Babrov [3] (красные окружности) и Séoudi et al. [5] (треугольники). Значения из базы данных HITRAN $[8,9]$ показаны сплошной линией. На нижней панели приведены метрологическая совместимость между коэффициентами двух изотопологов, измеренных в настоящей работе (синие столбики), и метрологическая совместимость результатов настоящей работы и работы [7] (штрихованные серые столбики). Расчет выполнен для доверительной вероятности $95 \%$. 
ях, но асимметрии формы линии, указывающей на сверхтонкую структуру, обнаружено не было. Коэффициент уширения для $R_{2}(7)$ совпадает с нашим значением в пределах погрешности.

\section{Сдвиги}

Коэффициенты сдвига $\delta$ являются коэффициентами наклона зависимостей позиций максимумов линий (максимумов контуров Фойгта) от давления. При ближайшем рассмотрении этих зависимостей (рис. 4) мы заметили, что изменение апертуры с 0.8 до $1 \mathrm{~mm}$ (табл. 1) вызывает (или убирает) систематический сдвиг $\sim 10^{-3} \mathrm{~cm}^{-1}$. Апертура была изменена для увеличения сигнала и соответственно отношения сигнал/шум, однако из-за неидеальной юстировки спектрометра это привело к сдвигу волновых чисел. Очевидно, что одновременная подгонка значений волновых чисел для всех давлений приведет к систематической ошибке. Для того чтобы ее избежать, мы раздельно подогнали прямыми линиями данные для различных апертур. При этом необходимо использовать распределения Стьюдента [19] для корректного определения погрешностей. Мы не получили значительного отличия полученных значений в этих двух группах и усреднили их, используя инструментальные веса $^{3}$.

На следующем этапе коэффициенты сдвига для изотопологов были сравнены между собой. Как и с коэффициентами уширения, статистического различия между ними замечено не было. Это позволило их усреднить, уменьшив погрешность значений. Полученные результаты показаны кружками в верхней части рис. 5 и перечислены в табл. 2. Большинство коэффициентов сдвига в обертоне $\mathrm{HBr}$ - батохромные (красные), лишь сдвиги для линий $R_{2}(0)$ и $P_{2}(2)$ - гипсохромные (синие). Метрологическая совместимость для различных изотопологов показана на нижней панели рис. 5: при доверительной вероятности 95\% эта совместимость находится в интервале $[-1,1]$ для всех $m$ без исключения.

Вакуумные волновые числа $v_{0}^{\text {fit5}}$, полученные из подгонки максимумов линий в спектрах, записанных с апертурой $0.8 \mathrm{~mm}$, превосходят данные из HITRAN примерно на $0.003 \mathrm{~cm}^{-1}$. Наиболее вероятно, что это является следствием неидеальной юстировки прибора. К сожалению из-за использования фильтров в спектрах отсутствовали какие-либо референсные линии, по которым можно было бы откалибровать прибор по частоте (например, линии остатков воды в приборе). Тем не менее было замечено, что разность $v_{0}^{\text {fit5 }}\left(\mathrm{H}^{79} \mathrm{Br}\right)-v_{0}^{\mathrm{fit}}\left(\mathrm{H}^{81} \mathrm{Br}\right)$ не зависит от абсолютных значений и отличается от аналогичных разностей в базе данных HITRAN для каждого $m$ на одно и то же число $2.89(6) \cdot 10^{-4} \mathrm{~cm}^{-1}$.

\footnotetext{
${ }^{3}$ Согласно Origin, если значение какой-либо величины имеет погрешность $\Delta x$, то при определении инструментального веса при различных подгонках, в том числе при усреднении, значение берется с весом, обратно пропорциональным дисперсии, $1 / \Delta x^{2}$.
}

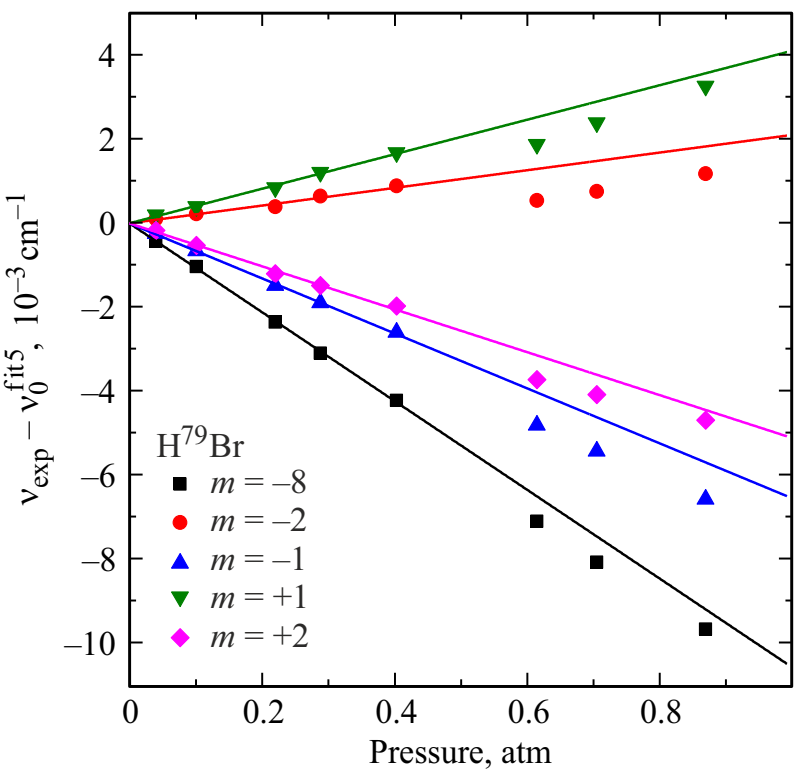

Рис. 4. Разность экспериментального максимума линии, $v_{\exp }$, и вакуумного волнового числа, $v_{0}^{\text {fit5 }}$, полученного из подгонки первых пяти точек, как функция давления для некоторых линий $\mathrm{H}^{79} \mathrm{Br}$. Индивидуальные погрешности не превышают размеров символов. Точки, соответствующие низким давлениям, получены из спектров, измеренных с апертурой $0.8 \mathrm{~mm}$; выше 405 mbar апертура была увеличена до $1 \mathrm{~mm}$.

Волновые числа в HITRAN для различных изотопологов и полос вычислены Coxon и Hajigeorgiou [20] на основе экспериментальных данных только для $\mathrm{H}^{79} \mathrm{Br}$ и $\mathrm{D}^{79} \mathrm{Br}$. Это дает нам основания полагать, что волновые числа линий в области первого обертона в базе данных требуют проверки, особенно для изотополога $\mathrm{H}^{81} \mathrm{Br}$.

Колебательный коэффициент самосдвига $\delta_{\text {vib }}$ может быть получен из зависимости колебательного волнового числа $v_{\text {vib }}$ от давления. Последнее было получено аппроксимацией колебательно-вращательных волновых чисел линий в полосе обертона для каждого давления полиномом шестой степени (учитывая вращательные постоянные для B, D и Н). Полученные колебательные коэффициенты самосдвига - гипсохромные (синие), что несколько неожиданно: $\delta_{\text {vib }}\left(\mathrm{H}^{79} \mathrm{Br}\right)=+2(5) \cdot 10^{-4} \mathrm{~cm}^{-1} \mathrm{~atm}^{-1}$, $\delta_{\text {vib }}\left(\mathrm{H}^{81} \mathrm{Br}\right)=+5(3) \cdot 10^{-4} \mathrm{~cm}^{-1} \mathrm{~atm}^{-1}$, с усредненным значением $\delta_{\text {vib }}=+4(3) \cdot 10^{-4} \mathrm{~cm}^{-1} \mathrm{~atm}^{-1}$. Несмотря на

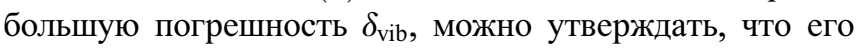
значение на порядок величины меньше, чем сдвиг колебательно-вращательных линий.

Также на рис. 5 показаны коэффициенты самосдвига других галоидоводородов. Самосдвиги $\mathrm{HCl}$ [21] часто меняют знак в $P$-ветви и сильно отклоняются от монотонного поведения, но остаются батохромными в $R$-ветви, начиная с $R_{2}(1)$. Значения для $\mathrm{HCl}$ в $R$-ветви практически совпадают со значениями для $\mathrm{HBr}$ и $\mathrm{HI}$. Видно, что коэффициенты сдвига линий $\mathrm{HBr}$ и $\mathrm{HI}$ [22] имеют сходные значения и зависимость от вращательно- 

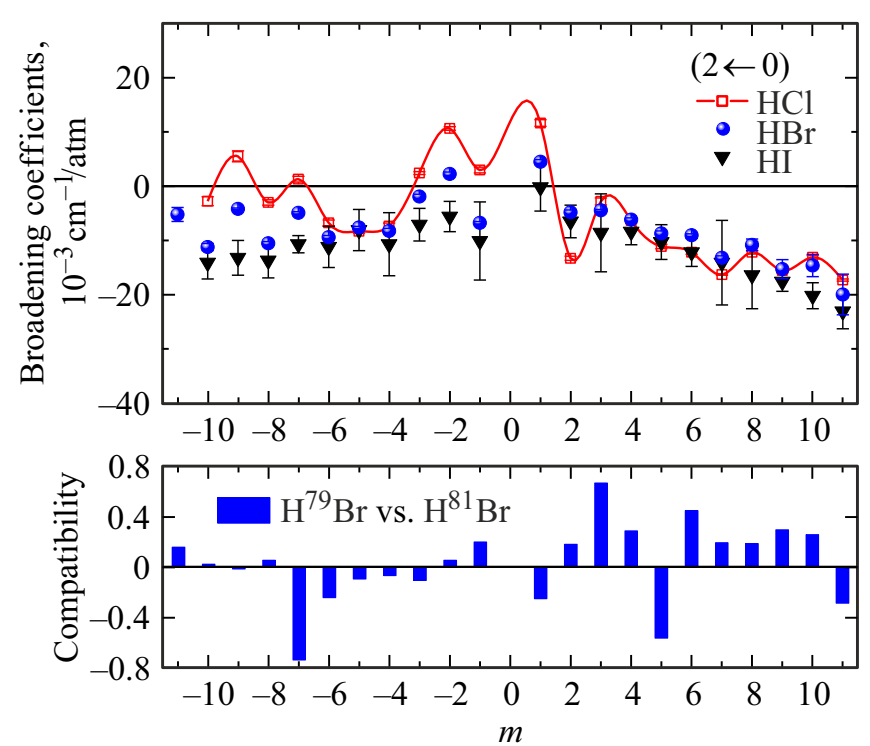

Рис. 5. Коэффициенты самосдвига в полосе первого обертона различных галоидоводородов. Значения для $\mathrm{HBr}$ (настоящая работа) показаны кружками. Данные для $\mathrm{HCl}$ (квадраты) взяты из [21], для НІ (треугольники) - из [22]. На нижней панели представлены совместимости коэффициентов сдвига для $\mathrm{H}^{79} \mathrm{Br}$ и $\mathrm{H}^{81} \mathrm{Br}$.

го состояния, сдвиги НІ при этом несколько сильнее и исключительно батохромные. Самосдвиг НF так сильно отличается от значения для более тяжелых галоидоводородов, что мы опустили его из рис. 5, поскольку он сильно перегрузил бы его. Читателям, интересующимся самосдвигом в HF, рекомендуем работы [23] (фундаментальная полоса) и [24] (первый обертон).

\section{Интенсивности линий}

Наших данных было достаточно, чтобы оценить интенсивности линий. Зависимости интегральной оптической плотности от давления имеют хорошую линейность: коэффициент детерминации $R^{2}>0.9995$ для большинства линий. Коэффициенты наклона этих зависимостей - это интенсивности линий при единичном давлении, $S_{v}^{P}$. В базе данных HITRAN используются значения $S_{v}^{N}$ - интенсивности линий на молекулу при стандартной температуре $296 \mathrm{~K}$ [25]. Оба значения приведены в табл. 3. Отметим также, что из определения $S_{v}^{P}$ следует его независимость от систематических сдвигов в значениях давления.

Сравнение интенсивностей линий в первом обертоне $\mathrm{H}^{79} \mathrm{Br}$ представлено на рис. 6. Наши данные заметно (но не драматично) превышают результаты предыдущих исследований. Наблюдается удовлетворительное согласие с интенсивностями из [12]. Интенсивности HITRAN [10] для первого обертона в значительной степени основаны на работе [12], поэтому они тоже сравнимы с нашими для большинства линий (хотя и на границе совместимо- сти), принимая погрешность в 5\%, заявленную в базе данных и соответствующую доверительной вероятности 95\%. Как видно из верхней панели рисунка, значения интенсивности, полученные из диодных лазерных измерений для двух линий [6], весьма близки к нашим, но их заявленная погрешность так мала, что метрологическая совместимость (2) сильно превышает единицу. Интенсивности линий Rao и Lindquist [4] и Bernage и Niay [11] на 15-30\% меньше, чем наши значения. Подобно нам авторы [10] отмечают, что их анализ указывает на занижение интенсивностей из $[4,11]$.

Поскольку значения для коэффициентов уширения в данной работе не показывают никаких аномалий (рис. 3), то сильное отклонение показаний датчика давления от истинных может быть исключено. Одним из объяснений, почему наши значения больше, чем литературные, может быть недооценка вклада крыльев линий при процедуре интегрирования, используемой в предыдущих работах $[3,12]$. Процедуры подгонки свободны от такого эффекта, так как форма линий учитывает крылья полностью. Тем не менее мы остерегаемся назвать это основной причиной. Проведение новых измерений
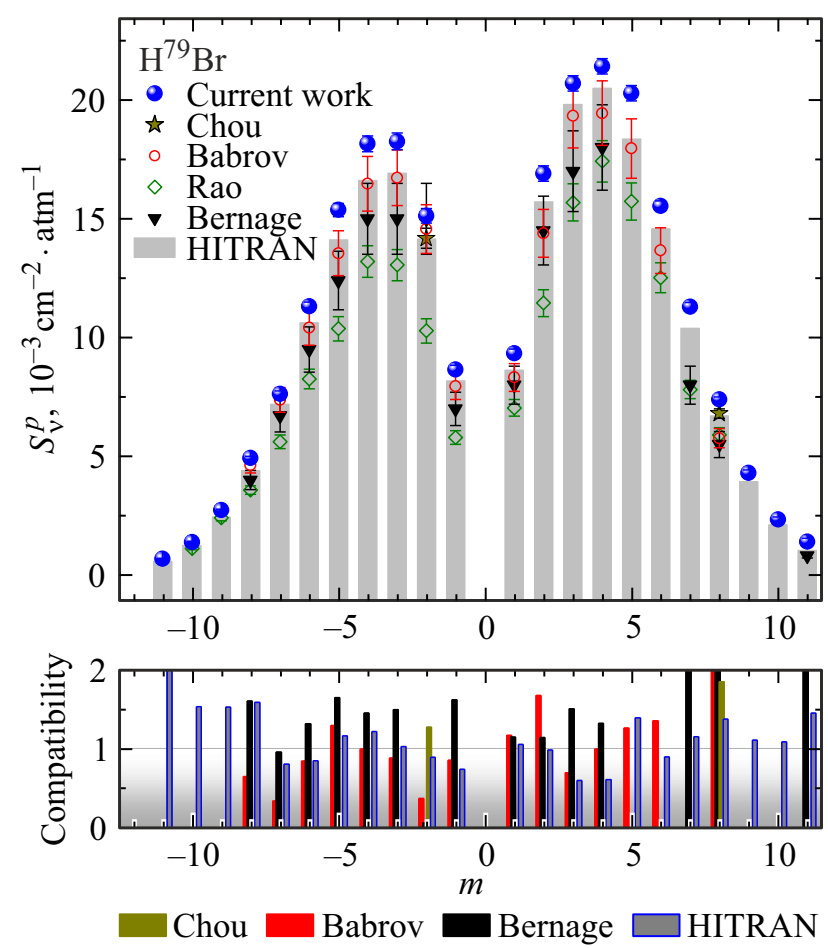

Рис. 6. Интенсивности линий в первом обертоне $\mathrm{H}^{79} \mathrm{Br}$. Данные из настоящей работы показаны синими кружками. Для сравнения приведены данные из других источников: Chou et al. [6] (звездочки), Babrov [3] (окружности), Rao et al. [4] (ромбы), Bernage et al. [11] (треугольники), база данных HITRAN [8] (серые столбцы). На нижней панели приведена метрологическая совместимость литературных значений с результатами данной работы. Опущена совместимость с данными Rao et al. [4], приведшая бы к загромождению рисунка в силу своей величины. 
Таблица 3. Интенсивности линий $S_{v}^{P}\left(\mathrm{~cm}^{-2} \mathrm{~atm}^{-1}\right) \mathrm{H}^{79} \mathrm{Br}$ и $\mathrm{H}^{81} \mathrm{Br}$ в первом обертоне при $297 \mathrm{~K}$ и естественном содержании изотопологов; также приведены интенсивности линий $S_{v}^{N}$ в единицах $\left(\mathrm{cm} \mathrm{molec}^{-1}\right)$, используемых в базе данных HITRAN (обозначения из [25]); в скобках указаны погрешности, соответствующие доверительной вероятности $95 \%$

\begin{tabular}{|c|c|c|c|c|}
\hline \multirow[b]{2}{*}{$m$} & \multicolumn{2}{|c|}{$\mathrm{H}^{79} \mathrm{Br}$} & \multicolumn{2}{|c|}{$\mathrm{H}^{81} \mathrm{Br}$} \\
\hline & $\begin{array}{c}S_{v}^{P} \\
10^{-3} \mathrm{~cm}^{-2} \mathrm{~atm}^{-1}\end{array}$ & $\begin{array}{c}S_{v}^{N} \\
10^{-22} \mathrm{~cm} \mathrm{molec}^{-1}\end{array}$ & $\begin{array}{c}S_{v}^{P}, \\
10^{-3} \mathrm{~cm}^{-2} \mathrm{~atm}^{-1}\end{array}$ & $\begin{array}{c}S_{v}^{N}, \\
10^{-22} \mathrm{~cm} \mathrm{molec}^{-1}\end{array}$ \\
\hline-11 & $0.68(2)$ & $0.274(7)$ & $0.65(2)$ & $0.264(7)$ \\
\hline-10 & $1.39(3)$ & $0.563(10)$ & $1.35(2)$ & $0.546(8)$ \\
\hline-9 & $2.73(4)$ & $1.10(2)$ & $2.65(4)$ & $1.07(2)$ \\
\hline-8 & $4.93(8)$ & $1.99(3)$ & $4.82(8)$ & $1.95(3)$ \\
\hline-7 & $7.6(1)$ & $3.09(5)$ & $7.4(1)$ & $3.01(5)$ \\
\hline-6 & $11.3(2)$ & $4.58(8)$ & $11.0(2)$ & $4.44(8)$ \\
\hline-5 & $15.4(3)$ & $6.2(1)$ & $14.9(3)$ & $6.0(1)$ \\
\hline-4 & $18.2(4)$ & $7.4(2)$ & $17.7(3)$ & $7.2(1)$ \\
\hline-3 & $18.3(4)$ & $7.4(2)$ & $17.9(4)$ & $7.2(2)$ \\
\hline-2 & $15.1(3)$ & $6.1(1)$ & $14.8(3)$ & $6.0(1)$ \\
\hline-1 & $8.7(2)$ & $3.51(7)$ & $8.5(2)$ & $3.45(7)$ \\
\hline 1 & $9.3(2)$ & $3.78(8)$ & $9.1(2)$ & $3.67(7)$ \\
\hline 2 & $16.9(3)$ & $6.8(2)$ & $16.4(3)$ & $6.6(1)$ \\
\hline 3 & $20.7(3)$ & $8.4(2)$ & $20.0(3)$ & $8.1(1)$ \\
\hline 4 & $21.4(3)$ & $8.7(2)$ & $20.9(3)$ & $8.4(1)$ \\
\hline 5 & $20.3(4)$ & $8.2(2)$ & $19.9(3)$ & $8.0(1)$ \\
\hline 6 & $15.5(2)$ & $6.29(9)$ & $15.2(2)$ & $6.14(8)$ \\
\hline 7 & $11.3(2)$ & $4.57(7)$ & $11.0(2)$ & $4.47(7)$ \\
\hline 8 & $7.40(10)$ & $2.99(4)$ & $7.2(1)$ & $2.93(5)$ \\
\hline 9 & $4.3(1)$ & $1.74(5)$ & $4.3(1)$ & $1.75(5)$ \\
\hline 10 & $2.34(10)$ & $0.95(4)$ & $2.2(2)$ & $0.90(6)$ \\
\hline 11 & $1.4(2)$ & $0.57(6)$ & $1.33(9)$ & $0.54(4)$ \\
\hline
\end{tabular}

интенсивностей безусловно поможет пролить свет на этот вопрос.

\section{Заключение}

В данной работе получен набор коэффициентов самоуширения и сдвига, а также интенсивностей линий в первом обертоне молекул $\mathrm{H}^{79} \mathrm{Br}$ и $\mathrm{H}^{81} \mathrm{Br}$. Отличие между столкновительными параметрами для разных изотопологов не обнаружено. Коэффициенты самоуширения находятся в прекрасном согласии с предыдущими измерениями $[6,7]$ (за исключением значения $P_{2}(2)$ из работы [6]). Кроме того, они имеют меньшую погрешность (около 1\% для линий в интервале $-10 \leq m \leq 8$ при доверительной вероятности 95\%). Заметим, что значения из HITRAN для $P$-ветви заметно отличаются от экспериментальных, поскольку они представляют лишь зеркальное отражение значений из $R$-ветви. Зависимость коэффициентов уширения от номера линий $m$ часто близка к симметричной, но, как мы видим (рис. 3), это не выполняется для $\mathrm{HBr}$.

Большинство коэффициентов сдвига в обертоне $\mathrm{HBr}$ являются батохромными (красными), хотя колебательный коэффициент сдвига - гипсохромный (синий). Погрешности коэффициентов сдвига для большинства линий в диапазоне $-10 \leq m \leq 8$ менее $10 \%$. Насколько нам известно, коэффициенты сдвига были определены впервые.

Найденные в данной работе интенсивности находятся в разумном согласии со значениями из $[3,6]$, так же как и из HITRAN [8], хотя несколько превосходят их (рис. 6). $\mathrm{y}$ нас нет однозначного объяснения этим несколько бо́льшим значениям. Возможно, это результат различия в учете крыльев линий при описании спектров определенными контурами и процедурой интегрирования. Дополнительные измерения безусловно могут оказаться полезными.

\section{Благодарности}

Эта статья посвящена нашему коллеге, учителю и другу Михаилу Олеговичу Буланину, который привил нам интерес к изучению спектроскопических свойств двухатомных молекул.

\section{Финансирование работы}

Работа выполнена при финансовой поддержке Немецкого научно-исследовательского общества (DFG), грант Мa 1845/7-1. 


\section{Конфликт интересов}

Авторы заявляют, что у них нет конфликта интересов.

\section{Список литературы}

[1] Hydrogen Bromide Market - Global Industry Analysis, Size, Share, Growth, Trends, and Forecast, 2019-2027. Электронный ресурс. Режим доступа: https://www.transparencymarketresearch.com/ hydrogen-bromide-market.html

[2] Hydrogen Bromide Market: Global Industry Analysis and Forecast 2016 - 2022. Электронный ресурс. Режим доступа: https://www.persistencemarketresearch.com/market-research/ hydrogen-bromide-market.asp

[3] Babrov H.J. // J. Chem. Phys. 1964. V. 40. N 3. P. 831. doi 10.1063/1.1725213

[4] Rao B.S., Lindquist L.H. // Can. J. Phys. 1968. V. 46. N 24. P. 2739. doi 10.1139/p68-641

[5] Séoudi B., Henry A., Margottin-Maclou M. // J. Mol. Spectrosc. 1985. V. 112. N 1. P. 88. doi 10.1016/0022-2852(85)90194-8

[6] Chou S.-I., Baer D.S., Hanson R.K. // J. Mol. Spectrosc. 2000. V. 200. N 1. P. 138. doi 10.1006/jmsp.1999.8038

[7] Domanskaya A.V., Bulanin M.O., Kerl K., Maul C. // J. Mol. Spectrosc. 2009. V. 253. N 1. P. 20. doi 10.1016/j.jms.2008.09.010

[8] The HITRAN Database. Электронный ресурс. Режим доступа: https://hitran.org/

[9] Gordon I.E., Rothman L.S., Hill C., Kochanov R.V., Tan Y., Bernath P.F., Zak E.J. // JQSRT. 2017. V. 203. P. 3. doi 10.1016/j.jqsrt.2017.06.038

[10] Li G., Gordon I.E., Le Roy R.J., Hajigeorgiou P.G., Coxon J.A., Bernath P.F., Rothman L.S. // JQSRT. 2013. V. 121. P. 78. doi 10.1016/j.jqsrt.2013.02.005

[11] Bernage P., Niay P. // JQSRT. 1977. V. 18. N 3. P. 315. doi 10.1016/0022-4073(77)90062-0

[12] Babrov H.J., Shabott A.L., Rao B.S. // J. Chem. Phys. 1965. V. 42. N 12. P. 4124. doi 10.1063/1.1695905

[13] Li G., Asfin R.E., Domanskaya A.V., Ebert V. // Mol. Phys. 2018. V. 116. N 23-24. P. 3495. doi 10.1080/00268976.2018.1457805

[14] Domanskaya A.V., Asfin R.E., Kyuberis A.A., Ebert V. // JQSRT. 2019. V. 235. P. 209. doi 10.1016/j.jqsrt.2019.07.004

[15] Bulanin M.O., Domanskaya A.V., Kerl K. // J. Mol. Spectrosc. 2003. V. 218. N 1. P. 75. doi 10.1016/S0022-2852(02)00063-2

[16] Hartmann J.-M., Boulet C., Robert D. Collisional Effects on Molecular Spectra. Elsevier, 2008. doi 10.1016/B978-0-444-52017-3.X0001-5

[17] Joint Committee for Guides in Metrology. BIPM International vocabulary of metrology. Электронный ресурс. Режим доступа: https://www.bipm.org/en/publications/guides/vim.html

[18] Rothman L.S., Gamache R.R., Tipping R.H., Rinsland C.P., Smith M.A.H., Benner D.C., Toth R.A. // JQSRT.1992. V. 48. N 5-6. P. 469. doi 10.1016/0022-4073(92)90115-K

[19] NIST/SEMATECH e-Handbook of Statistical Methods. NIST/SEMATECH, 2013. doi 10.18434/M32189

[20] Coxon J.A., Hajigeorgiou P.G. // JQSRT. 2015. V. 151. P. 133. doi 10.1016/j.jqsrt.2014.08.028

[21] Li G., Serdyukov A., Gisi M., Werhahn O., Ebert V. // JQSRT. 2015. V. 165. P. 76. doi 10.1016/j.jqsrt.2015.06.021
[22] Hartmann J.M., Bouanich J.P., Boulet C., Bulanin M.O., Domanskaya A.V., Kerl K. // JQSRT. 2005. V. 95. N 2. P. 151. doi 10.1016/j.jqsrt.2004.10.002

[23] Pine A.S., Fried A. // J. Mol. Spectrosc. 1985. V. 114. N 1. P. 148. doi 10.1016/0022-2852(85)90344-3

[24] Guelachvili G., Smith M.A.H. // JQSRT. 1978. V. 20. N 1. P. 35. doi 10.1016/0022-4073(78)90004-3

[25] Šimečková M., Jacquemart D., Rothman L.S., Gamache R.R., Goldman A. // JQSRT. 2006. V. 98. N 1. P. 130. doi $10.1016 /$ j.jqsrt.2005.07.003 